\title{
Effects of engineered conserved dopamine neurotrophic factor-expressing bone marrow stromal cells on dopaminergic neurons following 6-OHDA administrations
}

\author{
JIAMING MEI and CHAOSHI NIU \\ Department of Neurosurgery, Anhui Provincial Hospital Affiliated to Anhui Medical University, \\ Anhui Province Key Laboratory of Brain Function and Brain Diseases, Hefei, Anhui 230001, P.R. China
}

Received January 23, 2014; Accepted September 19, 2014

DOI: $10.3892 / \mathrm{mmr} .2014 .2878$

\begin{abstract}
Numerous lines of evidence previously indicated that conserved dopamine neurotrophic factor (CDNF) has potential therapeutic value for Parkinson's disease (PD); however, this hypothesis remains controversial. In the present study, the therapeutic effects of engineered CDNF-expressing bone marrow stromal cells (CDNF-BMSCs) on dopaminergic (DA) neurons were evaluated in vivo. CDNF-BMSCs and control BMSCs were transplanted into the rat striatum and one week later, 6-hydroxydopamine (6-OHDA) was administered to induce neurotoxicity. It was discovered that intrastriatal transplantation of CDNF-BMSCs significantly reduced 6-OHDA-induced neurotoxicity in DA neurons with regard to behavioral recovery and tyrosine hydroxylase levels in the substantia nigra and striatum. These data therefore indicated that transplantation of engineered CDNF-BMSCs into the striatum may present a beneficial strategy for the treatment of PD.
\end{abstract}

\section{Introduction}

Parkinson's disease (PD) is the most common neurodegenerative disorder and for which no cure is currently available (1). PD is characterized pathologically by the selective and progressive loss of dopaminergic (DA) neurons of the substantia nigra pars compacta (SNc) (1). Despite significant developments in available therapies, including drug treatment and deep brain stimulation, these treatments are only able to relieve the movement-associated symptoms of PD, rather than its pathological progression (1). The development of treatments to prevent or

Correspondence to: Dr Chaoshi Niu, Department of Neurosurgery, Anhui Provincial Hospital Affiliated to Anhui Medical University, Anhui Province Key Laboratory of Brain Function and Brain Diseases, 17 Lujiang Road, Hefei, Anhui 230001, P.R. China E-mail: doctorstar@126.com

Key words: conserved dopamine neurotrophic factor, bone marrow stromal cells, dopaminergic neurons, substantia nigra, corpus striatum, Parkinson's disease rescue the pathological deterioration remains the ultimate goal in $\mathrm{PD}$ research.

Although the pathogenesis of PD remains elusive, numerous neurotrophic factors have been identified which protect and rescue DA neurons from numerous assaults in vitro and in vivo (2-4). Conserved dopamine neurotrophic factor (CDNF) is a novel neurotrophic factor for DA neurons and is a secreted protein containing eight conserved cysteine residues (5). Lindholm et al (5) demonstrated that CDNF was able to protect and rescue midbrain DA neurons following 6-hydroxydopamine (6-OHDA)-induced neurotoxicity in vivo, as well as facilitating the recovery of the resulting behavioral deficits. Subsequently, several studies have demonstrated that administration of CDNF into the brain protected DA neurons from injury, rescued dying DA neurons in mice and facilitated the recovery of behavioral deficits in nonhuman primate models of PD (5-8). However, the beneficial effects of CDNF administration were not sustained following its withdrawal, indicating a requirement for continuous CDNF administration to maintain its neuroprotective effects (6). However, chronic infusion is technically challenging in a clinical setting (7). A cell-mediated gene therapy has the potential to improve the therapeutic efficacy of CDNF administration (9).

Previous studies have indicated that mesenchymal stem cells (MSCs) have the capacity to differentiate into numerous non-mesenchymal cell types in vivo $(8,10)$ and migrate extensively throughout the adult animal $(11,12)$. In addition, studies have reported that bone marrow-derived cells migrate preferentially to the sites of brain injury, for example ischemia, in rats $(2,13)$. These characteristics suggest that bone marrow stromal cells (BMSCs) may be a potential vehicle for the delivery of therapeutic genes to the diseased brain. In the present study, BMSCs were transfected with CDNF complementary DNA (cDNA) and transplanted into the brains of model rats with 6-OHDA-induced PD by intrastriatal infusion. The potential neuroprotective effects of the transplanted CDNF-expressing BMSCs (CDNF-BMSCs) on DA neurons were investigated in vivo.

\section{Materials and methods}

Recombinant plasmid construction. Wistar rats were obtained from Anhui Provincial Hospital Research Center(Hefei, China). 
The total RNA of the CDNF gene was isolated from the rats (564 bases) using TRIzol reagent (Invitrogen Life Technologies, Carlsbad, CA, USA). The first-strand cDNAs were subsequently synthesized by reverse transcription (RT), followed by polymerase chain reaction (PCR) using the following primers: Forward, 5'-ACCATGCGGTGCATCAGTCCAACTGC-3' and reverse, 5'-GAGCTCCGTTTGGGGGTATATC-3'. The pEGFP-N1-CDNF construct was created by digestion by double restriction enzymes (BamHI and XhoI), ligation and transfection into E. coli DH5 $\alpha$-competent cells (China Science Institute of Shanghai Life Science College, Shanghai, China). Purified pEGFP-N1-CDNF recombinant plasmid was transfected into BMSCs using Lipofectamine ${ }^{\circledR} 2000$ (Life Technologies, Grand Island, NY, USA) as described previously (2). At $72 \mathrm{~h}$ following the transfection, the BMSCs were harvested ready for transplantation.

Experimental design. Four separate groups of rats received four intrastriatal transplants, followed by PD modeling induced by 6-OHDA lesioning at the ipsilateral striatum one week later. The groups were as follows: Group $1(n=16)$, sham operation of intrastriatal transplantation followed by saline injection into striatum; group $2(n=16)$, sham operation of intrastriatal transplantation followed by 6-OHDA (Sigma-Aldrich, St. Louis, MO, USA) lesioning at the ipsilateral striatum; group $3(n=16)$, intrastriatal BMSC transplantation followed by 6-OHDA lesioning at the ipsilateral striatum; group $4(n=16)$ intrastriatal CDNF-BMSC transplantation followed by 6-OHDA lesioning at the ipsilateral striatum. Rats were evaluated 2, 4 and 6 weeks following 6-OHDA lesioning. Six weeks following 6-OHDA lesioning, the rats were sacrificed for immunohistochemical analysis by $\mathrm{TH}$ immunostaining in $\mathrm{SNc}$ and striatum.

Animals and surgical procedures. A total of 64 female Sprague-Dawley rats weighing 200-250 g were obtained from the Anhui Provincial Hospital Research Center (Hefei, China). The animals received food and water ad libitum and were kept under controlled environmental conditions (12-h light/dark cycle, with light on between 7:00 and 19:00 h; room temperature, $21^{\circ} \mathrm{C}$ ). During all experimental procedures, rats were treated in accordance with the Guidelines for Animal Care and Use of the National Institutes of Health (Bethesda, MD, USA).

Surgical procedures were performed under chloral hydrate [300 mg/kg, intraperintoneal injection (i.p.)] and executed in a Kopf stereotaxic apparatus (Narishige, Tokyo, Japan). Animals received three unilateral stereotaxic injections of variable amounts of 6-OHDA (Sigma-Aldrich) in various locations within the left striatum using a 10- $\mu$ l Hamilton microsyringe driven by a microinfusion pump (Shanghai Precision and Scientific Instrument Co., Ltd., Shanghai, China). The injection rate was $1 \mu \mathrm{l} / \mathrm{min}$ and the cannula was left in place for an additional two minutes prior to being slowly retracted. The doses and coordinates used were selected based on a previous study (14). The coordinates ( $\mathrm{mm}$ ) of the surgical procedure were as follows: Anteroposterior (AP), +0.48; mediolateral (ML), \pm 3.0 ; dorsoventral (DV), $-5.6 /-4.3 /-3.5$ by injection of $\mathrm{pt}$ aequ 6-OHDA (total amount, $20 \mu \mathrm{g} / 6 \mu \mathrm{l}$ ) (15). All solutions were $0.02 \%$ ascorbic acid in $0.9 \%$ saline and 6-OHDA solutions were freshly prepared, kept on ice and protected from exposure to light to minimize variability and degradation of the toxin.

Rotational behavior. At 2, 4 and 6 weeks following 6-OHDA lesion, the rats were assessed by apomorphine (APO; Sigma-Aldrich)-induced rotational asymmetry over $30 \mathrm{~min}$. Rotational behavior was monitored in automated rotameter (Panlab; DL Naturegene Life Sciences, Inc.; Beijing; China) bowls following APO administration (1). The number of rotations to the ipsilateral side were counted for $30 \mathrm{~min}$ following intraperitoneal administration of APO $(0.5 \mathrm{mg} / \mathrm{kg})$.

\section{Histology}

Perfusion and tissue processing for histology. Six weeks following 6-OHDA lesion, the rats were sacrificed. The animals were deeply anesthetized by an intravenous injection of chloral hydrate $(300 \mathrm{mg} / \mathrm{kg})$ and transcardially perfused with $0.9 \%$ saline followed by $4 \%$ buffered formaldehyde (Beijing Zhongshan Golden Bridge Biotechnology Co., Ltd., Beijing, China). The brains were removed, blocked, immersed in an identical fixative for $24 \mathrm{~h}$ and placed in saline (Beijing Zhongshan Golden Bridge Biotechnology Co., Ltd.) with the addition of 30\% sucrose (Beijing Zhongshan Golden Bridge Biotechnology Co., Ltd.) until the block sank. The brains were subsequently sectioned coronally using a cryostat (RM2015; Leica Microsystems GmbH, Wetzlar, Germany) at a thickness of $50 \mu \mathrm{m}$. Sections were collected in sequence and two series were collected onto gelatin-coated slides. The sections were deparaffinized and rehydrated. Following the addition of $3 \% \mathrm{H}_{2} \mathrm{O}_{2}$, the sections were washed with phosphate-buffered saline (PBS; ZLI-9062; Beijing Zhongshan Golden Bridge Biotechnology Co., Ltd., Beijing, China), incubated in citrate buffer (0.1M, pH 5.8; Beijing Zhongshan Golden Bridge Biotechnology Co., Ltd.) and washed repeatedly with PBS for immunohistochemistry (14).

Immunohistochemistry. Sections were immersed in a solution of $10 \%$ normal goat serum and $1 \%$ bovine serum albumin (in PBS) (both purchased from Beijing Zhongshan Golden Bridge Biotechnology Co., Ltd.) for $1 \mathrm{~h}$. Alternate sections were incubated with anti-tyrosine hydroxylase (TH; 1:500; Sigma-Aldrich) overnight at $4^{\circ} \mathrm{C}$. The sections were subsequently incubated with biotinylated goat anti-mouse immunoglobulin G secondary antibody (dilution, 1:3,000; Beijing Zhongshan Golden Bridge Biotechnology Co., Ltd.) for $15 \mathrm{~min}$ at room temperature. Finally, sections were incubated in avidin-biotin-peroxidase complex (Beijing Zhongshan Golden Bridge Biotechnology Co., Ltd.) for $15 \mathrm{~min}$ at room temperature. The bound peroxidase molecules were visualized using 3,3'-diaminobenzidine (Sigma-Aldrich). Between incubations, the sections were washed with PBS multiple times. Each of the antibodies, as well as the avidin-biotin-peroxidase complex, were diluted with PBS. Sections were placed on gelatin-coated slides, dried overnight, dehydrated in ascending alcohols, cleared in Histo-clear and mounted with distrene, plasticiser and xylene (Beijing Zhongshan Golden Bridge Biotechnology Co., Ltd.). For the control experiments, antibodies were replaced with equal volumes of PBS and the procedure was performed as above. Control sections were immunonegative. 
Table I. Changes in the number of apomorphine-induced rotations per $30 \mathrm{~min}$ following intrastriatal CDNF-engineered BMSC transplantation and 6-OHDA intrastriatal induction.

Time-point following 6-OHDA induction

\begin{tabular}{lccc}
\cline { 2 - 4 } Group & 2 weeks & 4 weeks & 6 weeks \\
\hline 1: Control (sham-operation) & 0 & 0 & 0 \\
2: Vehicle + 6-OHDA & $36.83 \pm 4.97$ & $120.92 \pm 9.89$ & $218.33 \pm 6.37$ \\
3: 6-OHDA + BMSCs & $34.67 \pm 2.90^{\mathrm{a}}$ & $63.08 \pm 6.58^{\mathrm{b}}$ & $162.67 \pm 11.63^{\mathrm{b}}$ \\
4: 6-OHDA + CDNF-BMSCs & $32.83 \pm 4.69^{\text {cd }}$ & $23.42 \pm 4.56^{\text {be }}$ & $12.00 \pm 2.89^{\text {be }}$
\end{tabular}

${ }^{\mathrm{a}} \mathrm{P}<0.206,{ }^{\mathrm{b}} \mathrm{P}<0.001,{ }^{\mathrm{c}} \mathrm{P}<0.055$ vs. group $2 .{ }^{\mathrm{d}} \mathrm{P}<0.262,{ }^{\mathrm{e}} \mathrm{P}<0.001$ vs group 3 . CDNF, conserved dopamine neurotrophic factor; BMSCs, bone marrow stromal cells; 6-OHDA, 6-hydroxydopamine.

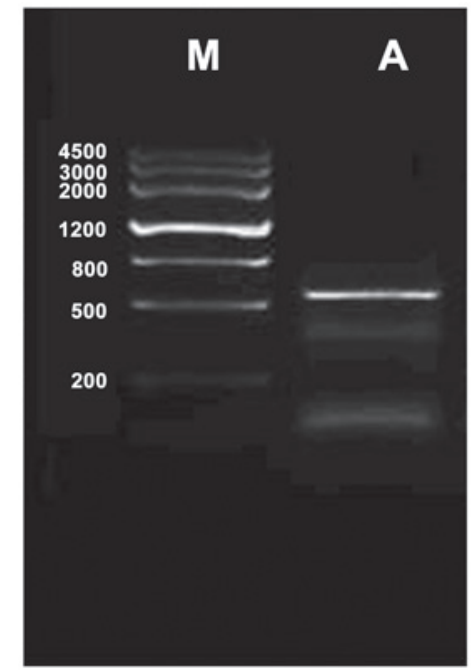

Figure 1. Electropherogram of CDNF gene PCR products of the pFastBacHTb-C1-CDNF construct. Lanes: M, marker; A, CDNF gene PCR products of pFastBacHTb-C1-CDNF construct. PCR, polymerase chain reaction; $\mathrm{CDNF}$, conserved dopamine neurotrophic factor.

\section{Morphological analysis}

SNc cell counts. The number of DA cells was determined using TH immunoreactivity. The total number of cells in the midbrain on each side of the brain was counted from ten sections in each animal (one section every five sections from the midbrain in sequence). Counts were made from comparable sections across the rostrocaudal extent of the ventral SNc. This region was the most clearly defined part of the nucleus and hence formed the focus of analysis. The present study did not aim to report on the total number of cells in the $\mathrm{SNc}$ of rats, instead, the number of cells in the nuclei of corresponding sections in various individual cases was compared. This strategy has been used in a study examining questions analagous to those addressed in the present study (16).

Striatal fiber density measurements. The optical densities of TH-immunoreactive fibers in the striatum were assessed using the image processing system of the Olympus BX51 (Olympus Corp., Tokyo, Japan). For each animal the optical density (OD) was measured at rostro-caudal levels according to the atlas of Paxinos and Watson (17) over the whole striatum: i) AP, 11.6;

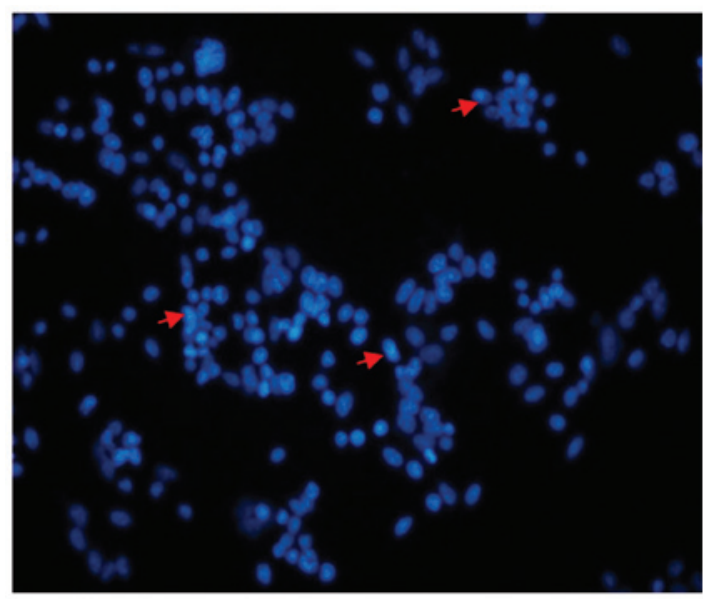

Figure 2. Recombinant pEGFP-N1-CDNF plasmid expression in bone marrow stromal cells determined by fluorescence microscopy. Blue fluorescence indicates successful plasmid recombination. Red arrows indicate recombinant pEGFP-N1-CDNF plasmid expression. CDNF, conserved dopamine neurotrophic factor. (Magnification, x200).

ii) AP, 11.0; iii) AP, 10.2; iv) AP, 20.3; v) AP, 20.9; vi) AP, 21.4 and vii) AP: 22.1 relative to the bregma. To estimate the specific TH staining density, the optical density readings were corrected for nonspecific background density, as measured from the completely denervated parts of the striatum in the animals with saline lesion. Data are presented as a percentage of the intact side.

Statistical analysis. Values are expressed as the mean \pm standard error of the mean of $n$ separate experiments. Statistical analysis between two groups was performed using Student's t-test and more than two groups were analyzed using analysis of variance, with SPSS 13.0 software (SPSS, Inc., Chicago, IL, USA). $\mathrm{P}<0.05$ was considered to indicate a statistically significant difference between values.

\section{Results}

Generating CDNF-expressing BMSCs for transplantation. Mouse CDNF cDNA was amplified by RT-quantitative PCR and cloned into pEGFP-N1 vectors for expressing CDNF (Fig. 1). The BMSC primary culture was identified 
Table II. TH immuno-positive expression following intrastriatal injection of CDNF-BMSCs and BMSCs six weeks following 6-OHDA administration.

\begin{tabular}{lcc}
\hline Group & $\begin{array}{c}\text { TH-positive cells in lesioned } \\
\text { substantia nigra }\end{array}$ & $\begin{array}{c}\text { Optical density value of TH-positive } \\
\text { fibers in lesioned striatum }\end{array}$ \\
\hline 1: Control & $616.67 \pm 26.67$ & $0.1963 \pm 0.0086$ \\
2: Vehicle + 6-OHDA & $128.00 \pm 11.86^{\mathrm{a}}$ & $0.0687 \pm 0.0123^{\mathrm{a}}$ \\
3: 6-OHDA + BMSCs & $371.33 \pm 28.31^{\mathrm{a}}$ & $0.1140 \pm 0.0125^{\mathrm{a}}$ \\
4: 6-OHDA + CDNF-BMSCs & $599.25 \pm 15.50^{\mathrm{bc}}$ & $0.1893 \pm 0.0690^{\mathrm{cd}}$ \\
\hline
\end{tabular}

${ }^{\mathrm{a}} \mathrm{P}<0.001,{ }^{\mathrm{b}} \mathrm{P}=0.063$ vs. group 1 ; ${ }^{\mathrm{c}} \mathrm{P}<0.001$ vs group 3 ; ${ }^{\mathrm{d}} \mathrm{P}=0.057$ vs. group 1 . $\mathrm{TH}$, tyrosine hydroxylase; CDNF, conserved dopamine neurotrophic factor; BMSCs, bone marrow stromal cells; OHDA, hydroxydopamine.

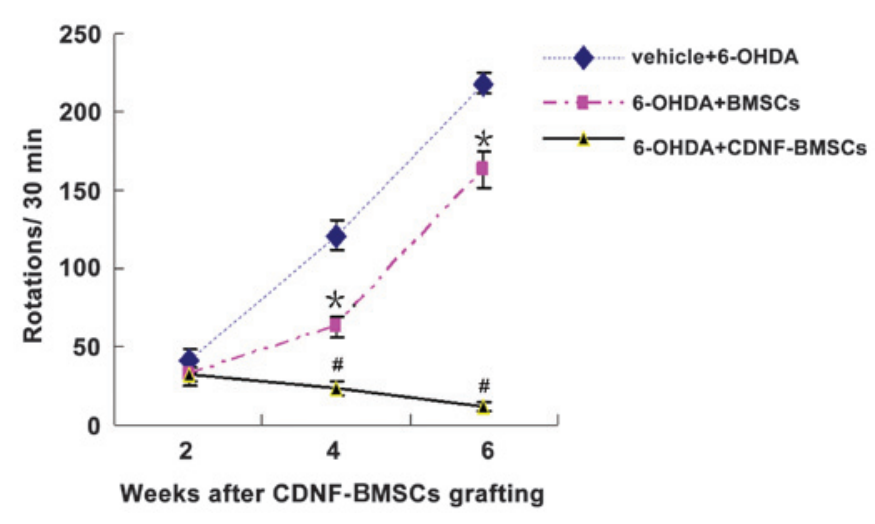

Figure 3. Effects of CDNF-BMSC transplantation on behavioral testing following 6-OHDA lesion. A significant reduction in apomorphine-induced rotations was observed following transplantation of CDNF-BMSCs or BMSCs into the rat striatum four and six weeks following 6-OHDA lesion. Compared with the group receiving 6-OHDA + BMSCs, rats receiving CDNF-BMSC transplantation showed significantly greater recovery four weeks following transplantation. ${ }^{*} \mathrm{P}<0.001$ vs. vehicle + 6-OHDA, ${ }^{"} \mathrm{P}<0.001$ vs. 6-OHDA + BMSCs. CDNF, conserved dopamine neurotrophic factor; BMSC, bone marrow stromal cell; 6-OHDA, 6-hydroxydopamine.

by fusiform cell shape and colony spread. The recombinant plasmid pEGFP-N1-CDNF was constructed and transfected into BMSCs and CDNF expression in BMSCs was confirmed by fluorescence microscopy (Fig. 2), the transfection efficiency was up to $56.8 \%$.

BMSC transplantation reduces APO-induced rotations. The results of the behavioral testing are exhibited in Table I and Fig. 3. In brief, two weeks following 6-OHDA administration, transplantation of BMSCs had significantly reduced APO-induced rotations compared with those of non-transplanted rats. Furthermore, the rats that had received a transplantation of CDNF-BMSCs had a significantly greater reduction in rotations at two, four and six weeks following lesion, compared to those of the rats which received BMSCs (Table I). This indicated that CDNF expression by transplanted BMSCs had significant neuroprotective effects against 6-OHDA-induced toxicity.

BMSC transplantation reduces 6-OHDA-induced loss of TH-positive fibers. Six weeks following 6-OHDA administration, immunohistochemistry was used to analyze TH-positive fibers in the striatum and differentiated DA neurons in the $\mathrm{SNc}$ (Fig. 4). The OD of TH immunoreactivity in the striatum of 6-OHDA-treated rats was significantly reduced (from $0.1963 \pm 0.0086$ to $0.0687 \pm 0.0123$ ). Transplantation of BMSCs significantly attenuated the reduction in TH-positive fibers $(\mathrm{P}<0.01)$. Transplantation of CDNF-BMSCs almost eliminated the 6-OHDA-induced loss of TH-positive fibers (OD, $0.1893 \pm 0.0690$ vs. $0.1963 \pm 0.0086$ ) (Table II).

In the 6-OHDA lesioned striatum group, 6-OHDA treatment caused the number of TH-positive neurons to decrease from $616.67 \pm 26.67$ (sham-operation group) to $128.00 \pm 11.86$ in the ipsilateral SNc, with somal collapse and unclear cytoarchitectonics (Fig. 5). The groups of rats which received BMSC or CDNF-BMSC transplantation exhibited a greater number of TH-positive cells in the SNc compared with that in the sham operation group $(\mathrm{P}<0.01)$. Furthermore, the number of TH-positive cells in the CDNF-BMSCs group (599.25 \pm 15.50$)$ was significantly greater than that in the BMSCs group (371.33 \pm 28.31 , P<0.01; Fig. 5).

\section{Discussion}

PD is a progressive movement disorder characterized pathologically by DA neuron degeneration in the SNc (1). Direct transfection with neurotrophic genes presented a potential therapeutic strategy. The ideal cellular vehicle for gene transfer into the brain was considered to be an autologous cell that does not disrupt brain circuitry (18-20). In previous studies, BMSCs have been successfully used as vehicles to deliver neurotrophin genes to the disease tissue $(3,21,22)$. In the present study, it was demonstrated that intrastriatal transplantation of CNDF-BMSCs significantly protected DA neurons against 6-OHDA-induced toxicity, resulting in behavioral recovery and attenuating the pathological degeneration of the substantia nigra (SN) and striatum. These data indicated the suitability of BMSCs as a vector for gene therapy and demonstrated that CDNF-expressing BMSCs as a subsidiary therapy may be beneficial in the treatment of PD.

DA axon terminal lesions, induced by intrastriatal injection of the neurotoxin 6-OHDA, have been developed as a tool for obtaining selective partial lesion of the nigrostriatal DA system in the rat (23-25). The stereotactic administration of 6-OHDA into the striatum initially induced direct toxic damage to the DA axons surrounding the injection site, followed by a gradual 

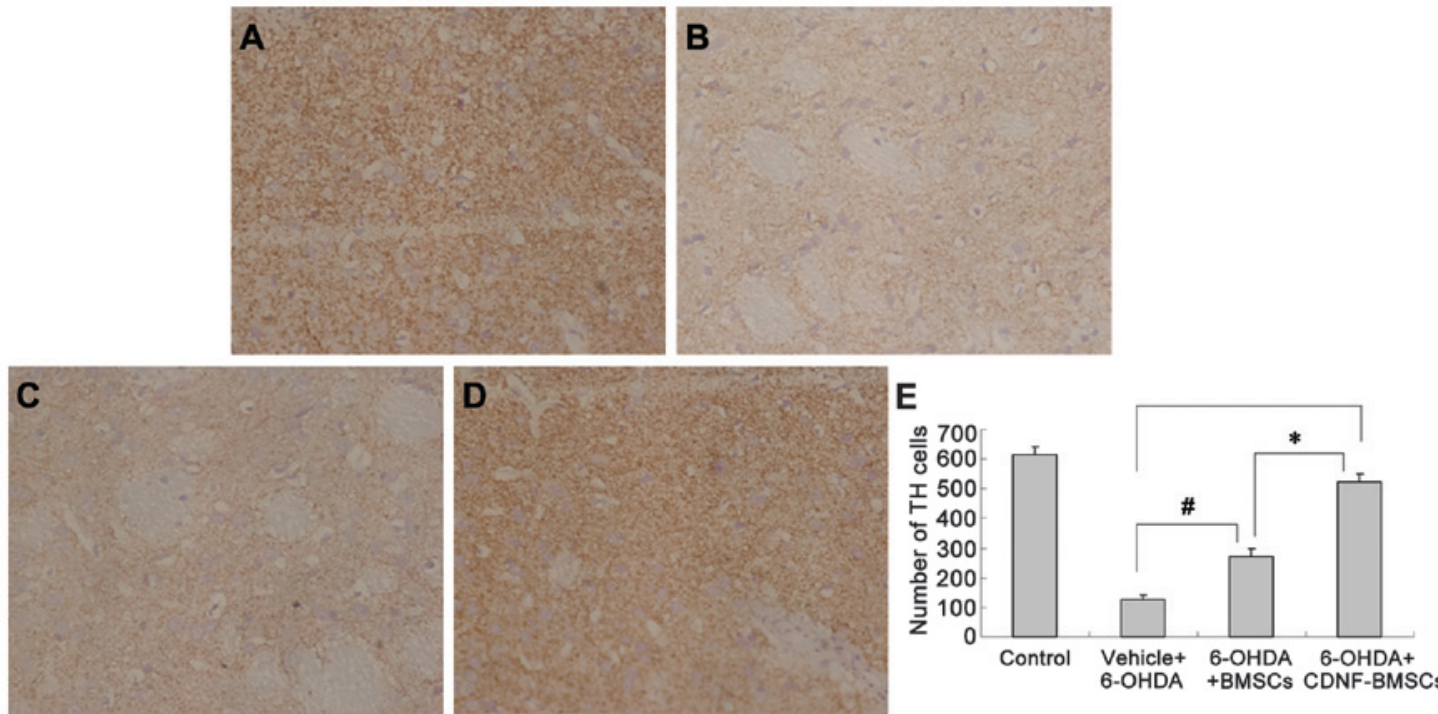

Figure 4. TH immunohistochemistry in the striatum following transplantation. TH-positive fiber density in the striatum was significantly increased in the 6-OHDA + BMSCs or 6-OHDA + CDNF-BMSCs groups compared with that in the control. (A) Control; (B) Vehicle + 6-OHDA; (C) 6-OHDA + BMSCs; (D) 6-OHDA + CDNF-BMSCs. (Magnification, $\mathrm{x} 400$ ). (E) Quantitative densitometry results. Values are presented as the mean \pm standard deviation. ${ }^{\prime \prime} \mathrm{P}<$, ${ }^{*} \mathrm{P}<$. TH, tyrosine hydroxylase; CDNF, conserved dopamine neurotrophic factor; BMSCs, bone marrow stromal cells; OHDA, hydroxydopamine. ${ }^{\prime \prime} \mathrm{P}<0.01$ and ${ }^{*} \mathrm{P}<0.001$.
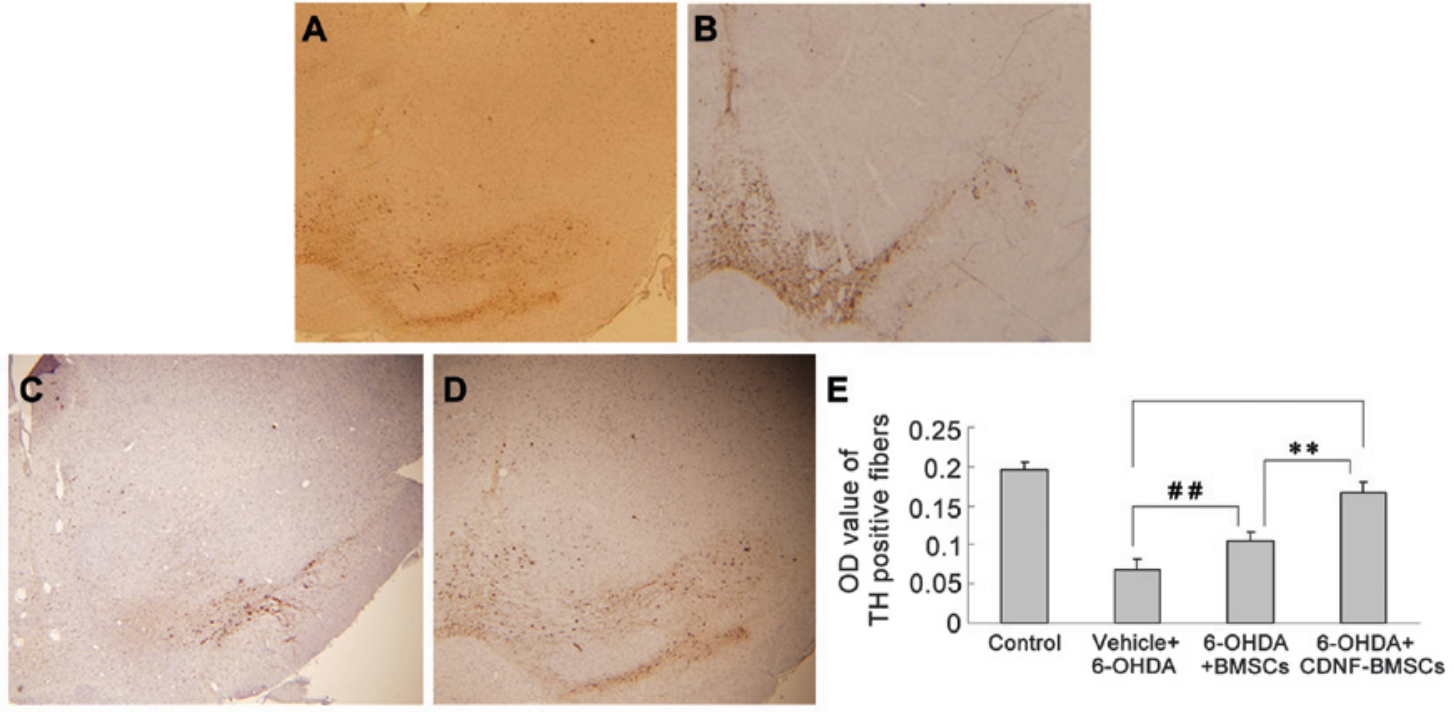

Figure 5. TH immunostaining in SNc following transplantation. The number of TH-positive cells in the SNc was significantly higher in the 6-OHDA + BMSCs and 6-OHDA + CDNF-BMSCs groups compared with that in the control. (A) Control; (B) 6-OHDA; (C) 6-OHDA + BMSCs; (D) 6-OHDA + CDNF-BMSCs. (Magnification, x50). (E) Quantitative densitometry results. Values are presented as the mean \pm standard deviation. ${ }^{\# \#} \mathrm{P}<$, ${ }^{\text {"**}} \mathrm{P}<$. TH, tyrosine hydroxylase; CDNF, conserved dopamine neurotrophic factor; BMSCs, bone marrow stromal cells; OHDA, hydroxydopamine, SNc, substantia nigra pars compacta; OD, optical density. ${ }^{\# \#} \mathrm{P}<0.01$ and ${ }^{* *} \mathrm{P}<0.001$.

loss of DA neurons in the ipsilateral SN (23). The functional effects induced by intrastriatal 6-OHDA administration depend not only on the total dose of injection, but additionally on the site of injection $(15,26,27)$. While a single high dose of 6-OHDA (20 g) failed to induce significant APO-induced rotation, pronounced behavioral symptoms were produced following distribution of an identical dose of toxin over three injection sites (27). In the present study, a three-site injection of 6-OHDA protocol was utilized, which induced an ideal PD model as indicated by the behavioral and pathological changes observed. It is advantageous to target the striatum, as opposed ot the SN, since intrastriatal induction may avoid damage to the SN by direct toxin lesion and mechanical insult (15). Therefore, rats with intrastriatal 6-OHDA lesions provide a model of progressive DA neuron degeneration, which is useful for the evaluation of potential neuroprotective therapies and additionally for the study of mechanisms underlying functional and structural recovery following damage to the nigrostriatal dopamine system.

The novel dopaminergic neurotrophic factor CDNF, a member of the highly conserved mesencephalic astrocyte-derived neurotrophic factor protein family, has been demonstrated to protect DA neurons from injury and rescue dying DA neurons following intrastriatal injection, as well as 
facilitate the recovery of behavioral deficits in 6-OHDA and 1-methyl-4-phenyl-1,2,3,6-tetrahydropyridine-induced rat models of PD (5-7). Further studies have indicated that chronic infusion of CDNF into the striatum by micro-pump, retrogradely transported from the striatum to the $\mathrm{SN}$, was able to prevent 6-OHDA-induced deficits in a rat model of PD (5-7). In general, intrastriatially injected CDNF was rapidly degraded in the brain and therefore, CDNF was unable to sustain its role in neuroprotection (7). Although chronic infusion of CDNF into the striatum has been used in animal models of PD, it is difficult to implement this process for clinical application. Therefore, gene therapy may provide an alternative therapeutic route.

Several studies have demonstrated thatbonemarrow-derived cells migrate preferentially to the sites of brain insults, including ischemia $(13,28)$ and diseased brain tissue in PD and Huntington's disease (29). Studies have shown that cDNA for glial cell line-derived neurotrophic factor transfected via BMSC vectors exerts neuroprotective and neurorestorative effects on DA neurons by engrafting into the striatum $(4,21)$. These characteristics indicated that the use of BMSCs as vehicles to deliver therapeutic genes into the sites of brain injury had the potential to improve functional deficits. In the present study, CDNF-BMSCs were delivered stereotactically into the striatum and exerted a significant neuroprotective effect. It was also revealed that the delivery of BMSCs produced significant neuroprotective effects. It was therefore hypothesized that the transplanted BMSCs may secrete CDNF or other neurotrophic factors. However, transplantation of CDNF-BMSCs produced a more significant neuroprotective effect than that of BMSCs, suggesting that the ectopic expression of CDNF by CDNFBMSCs was responsible for the differences observed. Greater therapeutic efficacy and safety issues represent a significant challenge in the development of practical applications of such therapies for PD in the future.

The neuroprotective effects of CDNF on DA neurons have gradually been established. BMSCs have the potential for multilineage differentiation and represent potentially useful vectors for the transfer of gene fragments to injury sites of the brain $(11-13,18,19)$. PD is characterized by the selective and progressive loss of DA neurons in the SN. Therefore, in the present study, BMSCs were utilized as a vector to deliver CDNF cDNA fragments into the SN, utilizing the characteristic of BMSCs of migrating to the brain injury site. A significant neuroprotective effect was revealed in a 6-OHDA-induced rat model following transplantation of CDNF-BMSCs into the striatum. Significant behavioral recovery and a reduction in the loss of TH-positive fibers in the $\mathrm{SNc}$ and striatum were observed. In conclusion, the results of the present study indicated that CDNF transfer via BMSC transplantation represent a potential therapeutic strategy for the treatment of PD.

\section{Acknowledgements}

The present study was supported by the Foundation of The Department of Education of Anhui Province (no. KJ2010B381), the Foundation of Natural Science of Anhui Province (no. 11040606Q11) and The National Natural Science Fund of China (no. 81100960).

\section{References}

1. Niu C, Mei J, Pan Q and Fu X: Nigral degeneration with inclusion body formation and behavioral changes in rats after proteasomal inhibition. Stereotact Funct Neurosurg 87: 69-81, 2009.

2. Su YR, Wang J, Wu JJ, Chen Y and Jiang YP: Overexpression of lentivirus-mediated glial cell line-derived neurotrophic factor in bone marrow stromal cells and its neuroprotection for the PC12 cells damaged by lactacystin. Neurosci Bull 23: 67-74, 2007.

3. Xiong N, Zhang Z, Huang J, et al: VEGF-expressing human umbilical cord mesenchymal stem cells, an improved therapy strategy for Parkinson's disease. Gene Ther 18: 394-402, 2011.

4. Park KW, Eglitis MA and Mouradian MM: Protection of nigral neurons by GDNF-engineered marrow cell transplantation. Neurosci Res 40: 315-323, 2001.

5. Lindholm P, Voutilainen MH, Laurén J, et al: Novel neurotrophic factor CDNF protects and rescues midbrain dopamine neurons in vivo. Nature 448: 73-77, 2007.

6. Airavaara M, Harvey BK, Voutilainen MH, et al: CDNF protects the nigrostriatal dopamine system and promotes recovery after MPTP treatment in mice. Cell Transplant 21: 1213-1223, 2012.

7. Voutilainen $M H$, Bäck S, Peränen J, et al: Chronic infusion of CDNF prevents 6-OHDA-induced deficits in a rat model of Parkinson's disease. Exp Neurol 228: 99-108, 2011.

8. Woodbury D, Schwarz EJ, Prockop DJ and Black IB: Adult rat and human bone marrow stromal cells differentiate into neurons. J Neurosci Res 61: 364-370, 2000.

9. Cheng L, Liu Y, Zhao H, Zhang W, Guo YJ and Nie L: Lentiviral-mediated transfer of CDNF promotes nerve regeneration and functional recovery after sciatic nerve injury in adult rats. Biochem Biophys Res Commun 18: 330-335, 2013.

10. Li JM, Zhu H, Lu S, et al: Migration and differentiation of human mesenchymal stem cells in the normal rat brain. Neurol Res 33: 84-92, 2011.

11. Brazelton TR, Rossi FM, Keshet GI and Blau HM: From marrow to brain: expression of neuronal phenotypes in adult mice. Science 290: 1775-1779, 2000.

12. Muñoz-Elias G, Marcus AJ, Coyne TM, Woodbury D and Black IB: Adult bone marrow stromal cells in the embryonic brain: engraftment, migration, differentiation, and long-term survival. J Neurosci 24: 4585-4595, 2004.

13. Eglitis MA, Dawson D, Park KW and Mouradian MM: Targeting of marrow-derived astrocytes to the ischemic brain. Neuroreport 10: 1289-1292, 1999.

14. Lee CS, Sauer H and Bjorklund A: Dopaminergic neuronal degeneration and motor impairments following axon terminal lesion by intrastriatal 6-hydroxydopamine in the rat. Neuroscience 72 : 641-653, 1996.

15. Kirik D, Rosenblad C and Björklund A: Characterization of behavioral and neurodegenerative changes following partial lesions of the nigrostriatal dopamine system induced by intrastriatal 6-hydroxydopamine in the rat. Exp Neurol 152: 259-277, 1998.

16. Lundblad M, Andersson M, Winkler C, Kirik D, Wierup N and Cenci MA: Pharmacological validation of behavioural measures of akinesia and dyskinesia in a rat model of Parkinson's disease. Eur J Neurosci 15: 120-132, 2002.

17. Paxinos G and Watson C: The Rat Brain in Stereotaxic Coordinates. 2nd edition, Academic Press, San Diego, 1986.

18. Rice CM and Scolding NJ: Autologous bone marrow stem cells - properties and advantages. J Neurol Sci 265: 59-62, 2008.

19. Benabdallah BF, Allard E, Yao S, et al: Targeted gene addition to human mesenchymal stromal cells as a cell-based plasma-soluble protein delivery platform. Cytotherapy 12: 394-399, 2010.

20. Schwarz SC and Schwarz J: Translation of stem cell therapy for neurological diseases. Transl Res 156: 155-160, 2010.

21. Wu J, Yu W, Chen Y, et al: Intrastriatal transplantation of GDNF-engineered BMSCs and its neuroprotection in lactacystin-induced Parkinsonian rat model. Neurochem Res 35: 495-502, 2010.

22. Moloney TC, Rooney GE, Barry FP, Howard L and Dowd E: Potential of rat bone marrow-derived mesenchymal stem cells as vehicles for delivery of neurotrophins to the Parkinsonian rat brain. Brain Res 1359: 33-43, 2010.

23. Haleagrahara N, Siew CJ and Ponnusamy K: Effect of quercetin and desferrioxamine on 6-hydroxydopamine (6-OHDA) induced neurotoxicity in striatum of rats. J Toxicol Sci 38: 25-33, 2013. 
24. Um JW, Park HJ, Song J, Jeon I, Lee G, Lee PH and Chung KC: Formation of parkin aggregates and enhanced PINK1 accumulation during the pathogenesis of Parkinson's disease. Biochem Biophys Res Commun 393: 824-828, 2010.

25. Zafar KS, Siddiqui A, Sayeed I, Ahmad M, Saleem S and Islam F: Protective effect of adenosine in rat model of Parkinson's disease: neurobehavioral and neurochemical evidences. J Chem Neuroanat 26: 143-151, 2003.

26. Richter F, Hamann M and Richter A: Moderate degeneration of nigral neurons after repeated but not after single intrastriatal injections of low doses of 6-hydroxydopamine in mice. Brain Res 1188: 148-156, 2008.
27. Przedborski S, Levivier M, Jiang H, Ferreira M, Jackson-Lewis V, Donaldson D and Togasaki DM: Dose-dependent lesions of the dopaminergic nigrostriatal pathway induced by intrastriatal injection of 6-hydroxydopamine. Neuroscience 67: 631-647, 1995.

28. Zou Z, Jiang X, Zhang W, Zhou Y, Ke Y, Zhang S and Xu R: Efficacy of Tyrosine Hydroxylase gene modified neural stem cells derived from bone marrow on Parkinson's disease - a rat model study. Brain Res 1346: 279-286, 2010.

29. Sadan O, Shemesh N, Cohen Y, Melamed E and Offen D: Adult neurotrophic factor-secreting stem cells: a potential novel therapy for neurodegenerative diseases. Isr Med Assoc J 11: 201-204, 2009. 